\title{
Cold ion beam in a storage ring as a platform for large-scale quantum computers and simulators: Challenges and directions for research and development
}

\author{
T. Shaftan ${ }^{1}$ and Boris B. Blinov $\circledast^{2}$ \\ ${ }^{1}$ National Synchrotron Light Source-II, Brookhaven National Laboratory, Upton, New York 11973, USA \\ ${ }^{2}$ Department of Physics, University of Washington, Seattle, Washington 98195, USA
}

(Received 28 October 2020; accepted 9 August 2021; published 13 September 2021)

\begin{abstract}
The purpose of this paper is to evaluate the possibility of constructing a large-scale storage-ring-type iontrap system capable of storing, cooling, and controlling a large number of ions as a platform for scalable quantum computing (QC) and quantum simulations. In such a trap, the ions form a crystalline beam moving along a circular path with a constant velocity determined by the frequency and intensity of the cooling lasers. In this paper, we consider a large leap forward in terms of the number of ions that serve as qubits in QC, from fewer than 100 available in state of the art linear ion-trap devices today to an order of $10^{5}$ crystallized ions in the storage-ring setup. This new trap design unifies two different concepts: the storage rings of charged particles and the linear ion traps used for QC and mass spectrometry. In this paper, we use the language of particle accelerators to discuss the ion state and dynamics. We outline the differences between the above concepts, analyze challenges of the large ring with a revolving chain of ions, and propose goals for the research and development required to enable future quantum computers with 1000 times more qubits than available today. The challenge of creating such a large-scale quantum system while maintaining the necessary coherence of the qubits and the high fidelity of quantum logic operations is significant. Performing analog quantum simulations may be an achievable initial goal for such a device. Quantum calculations and simulations of complex quantum systems will move forward both the fundamental science and the applied research. Nuclear and particle physics, many-body quantum systems, lattice gauge theories, and nuclear structure calculations are just a few examples in which a largescale quantum simulation system will become a very powerful tool to move forward our understanding of nature.
\end{abstract}

DOI: 10.1103/PhysRevAccelBeams.24.094701

\section{INTRODUCTION}

Quantum computers harness the power of quantum mechanics to perform a variety of important computational tasks significantly more efficiently than their classical counterparts [1]. Quantum simulators, on the other hand, can solve difficult problems in physics, chemistry, and biology exponentially faster than the most advanced classical supercomputers [2]. Both types of devices, the quantum computers and the quantum simulators, are similar in that they use quantum bits, or qubits, to encode information and perform calculations.

A physical qubit is a two-level quantum system that encodes information in its quantum state in a way that is similar to a classical bit, but in a much larger space: the

Published by the American Physical Society under the terms of the Creative Commons Attribution 4.0 International license. Further distribution of this work must maintain attribution to the author(s) and the published article's title, journal citation, and DOI.
Hilbert space, which for $N$ qubits has $2^{N}$ dimensions. This allows quantum devices to process vast amounts of information in parallel and to complete important computational tasks much faster than their classical counterparts [3].

Various physical systems are being actively investigated as potential qubit candidates, including single atoms [4] and ions [5], superconducting circuits [6], semiconductor devices [7], linear optical systems [8], nitrogen vacancies in diamonds [9], and others. The performance of these disparate systems varies widely in terms of the qubit state coherence, operational time scales, quantum gate fidelity, and scalability. Among these, superconducting qubits and trapped ions have emerged as leaders, due to their high operational fidelities and long coherence times. Current state of the art superconducting and ion-trap systems operate using relatively small numbers of physical qubits-fewer than 100 . Scaling up this number to hundreds of thousands of physical qubits, where running of practical and useful quantum computing (QC) algorithms with fully implemented error correction and fault-tolerant operations may become possible, is an outstanding challenge in the field of QC. 


\section{ION TRAPS AND STORAGE RINGS}

One of the most promising and advanced approaches in QC is based on ion traps [10,11]. Qubits encoded in the internal atomic level of individual ions have been shown to have extremely long coherence times [12], enabling highfidelity single-qubit and two-qubit gates $[13,14]$, qubit state initialization [15], and detection [16]. Ion traps are small electromagnetic devices that combine radiofrequency (rf) and static (dc) electric fields to produce conditions for charged particles to be confined. Laser cooling [17] is used to lower the trapped ions' kinetic energy so that the ions "crystallize" to form the so-called Coulomb crystals [18]. In a linear rf ion trap [19], the confining potential is elongated and the ions form a chain. The qubit is spanned by a pair of stable or metastable internal states in an individual ion. The qubit can be formed by the Zeeman [20] or hyperfine [21] sublevels in the ground state of the ion or by the combination of a ground state sublevel and a metastable excited state [22].

The motion of a single ion in an $\mathrm{rf}$ trap is accurately approximated by three-dimensional (3D) simple harmonic oscillator with secular frequencies $\omega_{x}, \omega_{y}$, and $\omega_{z}$, defined by the trap geometry, the ion's mass, and the driving field frequency (the typical range is $2 \pi \times 10-100 \mathrm{MHz}$ ) and amplitude (typically of order $1000 \mathrm{~V}$ ), resulting in secular frequencies in the range of $2 \pi \times 0.1-10 \mathrm{MHz}$. With $N$ ions in the trap, there are $3 \mathrm{~N}$ normal modes of harmonic oscillator motion.

To achieve high-fidelity qubit gates with trapped ions, the latter must be laser-cooled to very low temperatures, where the quantization of the ions' motional states in the harmonic oscillator potential becomes important. Specifically, the Lamb-Dicke limit [23] typically must be reached, in which the atom-laser interaction can only cause transitions that change the motional quantum state by 0 and \pm 1 , and all other transitions are strongly suppressed. The Lamb-Dicke parameter $\eta$, which can be expressed as a ratio of the ion's kinetic energy change due to absorption of a single photon to the energy quantum of the harmonic oscillator (in other words, the phonon energy), quantifies the coupling between the internal state transition and the motional states of an ion. If the condition $\eta \ll 1$ holds true, the single-photon recoil energy does not change the motional state of the ion, which is a requirement for high-fidelity quantum gates.

Ion cooling into the Lamb-Dicke limit is typically accomplished by Doppler cooling [24], followed by several stages of sideband cooling [23], although the second step may be replaced by Sisyphus cooling [25] or electromagnetically induced transparency (EIT) cooling [26]. For trapped particles, a single laser beam is sufficient for efficient Doppler cooling, provided that the $k$-vector of the Doppler cooling laser beam has significant components along all three $(x, y$, and $z)$ principal axes of the trap. The sideband cooling typically requires one or two laser beams (in the case of Raman sideband cooling [23]), while Sisyphus and EIT cooling both require two laser beams.

Historically, the development of linear ion traps used in contemporary QC stems from rf quadrupole mass spectroscopy devices [27]. The latter borderline with particle storage rings, with the subtle difference of keeping only a few ions frozen in place by intense laser cooling. It is insightful to look at the origin of both ion traps and ion rings, which, in a large part, comes from the pioneering work of W. Paul. In the 1950s, he had conceived the idea of the quadrupole mass spectrometer [28,29], which turned into a powerful device known today as the Paul trap. About a decade later, he proposed a modification of the same spectrometer, titled "Plasmabetatron" [30] according to the ringlike shape and superimposed electric and magnetic fields, which evolved into the PauL laser cooling acceleration system (PALLAS) radiofrequency quadrupole (RFQ) ring [31-35] in the 1990s. Indeed, both concepts, cross compared in our paper, stem from the ion mass spectrometry; however, the ion traps evolved far toward housing low-temperature ion crystals, suitable for carrying out quantum computations. One objective of our paper is to show that the other evolutionary branch of mass spectrometry, i.e., ion rings, holds the promise of being used as a quantum computation device with a large capacity of qubits [36-38].

An alternating gradient synchrotron or an RFQ ring, as a rule, are both of substantially larger size than the typical ion trap and, therefore, are capable of storing much larger numbers of charged particles, with up to $10^{10}$ particles orbiting on a ringlike trajectory and being bunched by an $\mathrm{rf}$ system [39]. When cooled to a sufficiently low temperature, where the mutual repulsion energy is greater than the ions' motional energy, an ion beam becomes "crystallized" and the crystal's type is determined by the combination of the density of ions and the strength of machine focusing. As the ion density increases, ions form: first, a one-dimensional (1D) chain, then a two-dimensional (2D) zig-zag, and finally a 3D "shell" structure. Ions in a crystal interact with each other through Coulomb repulsion, which, in terms of the normal modes of motion, can be described as phonons in various frequencies and degrees of freedom [40-43].

Doppler laser cooling in a storage ring [44] may be realized by two counterpropagating laser beams. Scanning the laser frequency, one can both probe and cool the ion beam while recording fluorescence with a photomultiplier in photon counting mode or using a single-photon counting camera. The techniques developed in experiments with crystalline beams enable injection, storage, acceleration, cooling, and control over hundreds of thousands of ions in a storage ring.

One of the issues limiting the scaling up of the trapped ion quantum computers (and, for that matter, all quantum computers) is the decoherence of qubit states, which sets a limit for the length of the working cycle of the computers. 
Quantum error correction (QEC) techniques include developing redundant chains of qubits with correction algorithms. However, in the present-day noisy intermediate scale quantum devices, QEC cannot yet be implemented. With the significantly larger number of qubits in a storagering device, it may be feasible to demonstrate a fully faulttolerant QC with QEC.

A traditional rf ion trap can hold at most $\sim 100$ ions in a stable linear chain [45], so scaling up the number of qubits in a trapped ion device requires some modifications to either the trap structure or the architecture of the quantum processor. Several proposals already exist for producing large-scale QC devices based on trapped ions. One such proposal, called a quantum charge-coupled device (QCCD) [46], is based on interacting ion traps, where multiple set of qubits are stored and moved around the (very complex) trap structure to accomplish the state preparation, quantum logic gates, and qubit detection. Several important elements of the QCCD architecture have been demonstrated [47-49] that serve as a proof of principle for chip-scale trapped-ion QC, including a recent demonstration of parallel qubit operations in a QCCD device [50].

Another proposed architecture, called a modular universal scalable trapped ion quantum computer, uses multiple nodes formed by small-scale ion trap devices linked by a photonic quantum interface [51]. Each node is a simple linear ion trap holding a small (10-30) number of qubits. The photonic links allow scaling the system up by adding the nodes and without increasing the complexity of the ion traps themselves.

While both of the above architectures are very promising, both rely on small building blocks, and the scaling is achieved by putting many such blocks together into a large, distributed system with multiple lasers and electronic controls associated with each single block. It is of great interest to understand if there are other systems capable of storing, cooling, and controlling a large $\left(10^{5}\right)$ number of ions. The future of QC might rest with distributed systems of moving ions. In contrast to the conventional ion traps, where the ions are stationary and the quantum logic gates are enabled by laser beams focused on individual ions using, for example, multichannel acousto-optic modulators (AOMs) [52], the ringlike computer will rely on moving ion strings, with ions crossing the interaction region one by one, and quantum gates controlled by a finite set of fixed laser beams, shaped in the time domain by AOMs or the electro-optic modulators.

In this paper, we attempt to address both of those requirements-i.e., (a) the large number of ions, bringing out the notion of a crystalline ion beam instead of a string of few tens of ions and (b) the fact that the ions are in motion. We compare a conventional linear ion trap quantum computer setup to a storage ring of crystalline ions, presenting their essential differences, outlining associated challenges, and pointing out directions of future $R \& D$ to enable a large-scale QC in a storage ring. In contrast to Ref. [38], where the authors in general terms shared the idea of using storage rings with crystalline beams as a quantum computer, we evaluate specific objectives ahead of the ring designers, assess their level of severity and propose mid-term $R \& D$ goal to make the first step along long path from the quantum computer on a modern ion trap to the large-scale processor on a future circular machine. We discuss the realistic system parameters, required crystal temperatures, propose the specific choice of good qubit candidates (singly charged ions of calcium and barium), and discuss the quantum gate implementations, and protocols for keeping track of individual qubits in the 100000 moving ion chain.

\section{GENERAL FORMULATION OF THE PROBLEM}

For concreteness, we consider an optical qubit in $\mathrm{Ca}^{+}$ and $\mathrm{Ba}^{+}$ions, which is formed by the ground state $\left(S_{1 / 2}\right.$, labeled $|g\rangle$ in the following discussion) and a metastable excited state $\left(D_{5 / 2}\right.$ labeled $\left.|e\rangle\right)$, also called the "shelved state," of the valence electron in these ions. The choice of ions is motivated by their distinctively different atomic mass and optical properties, covering a wide range of ions species suitable for use in QC. The relevant properties of these ions are summarized in Table 1.

Both ions species have been used in a variety of quantum computation protocols [21,22], and thus form a useful testbed for our study. Throughout this paper, we use a simple model of the ion states described in Fig. 1(a). Here, the yellow arrow represents the qubit transition, corresponding to single-qubit quantum gates, whereas the blue and red arrows are the spectral sidebands, which may be used for sideband cooling and multiqubit entangling gates. The spectral sidebands are caused by the harmonic motion of ions in the trap whose frequency (referred to as the phonon frequency in Fig. 1, i.e., $|\mathrm{g}, 0\rangle$ to $|g, 1\rangle$ transition) is of order $10^{6} \mathrm{~Hz}$, while the qubit transition frequency $|g\rangle$ to $|e\rangle$ is of order $10^{14} \mathrm{~Hz}\left(\sim 3.78 \times 10^{14} \mathrm{~Hz}\right.$ in $\mathrm{Ca}^{+}$or $\sim 1.70 \times 10^{14} \mathrm{~Hz}$ in $\left.\mathrm{Ba}^{+}\right)$. The machine geometry is shown schematically in Fig. 1(b). Similar to the linear ion trap, we consider strictly a $1 \mathrm{D}$ geometry of the ion beam, where the

TABLE I. Comparison between two types of ions for QCs.

\begin{tabular}{lcc}
\hline \hline Species & $\mathrm{Ca}^{+}[22]$ & $\mathrm{Ba}^{+}[21]$ \\
\hline Isotope & 40 & 138 \\
Lifetime of shelved state & $1 \mathrm{~s}$ & $32 \mathrm{~s}$ \\
Cooling transition & $397 \mathrm{~nm}$ & $493 \mathrm{~nm}$ \\
Qubit transition & $792 \mathrm{~nm}$ & $1762 \mathrm{~nm}$ \\
Typical transverse & $2 \pi \times 2.5 \mathrm{MHz}$ & $2 \pi \times 1.0 \mathrm{MHz}$ \\
$\quad$ secular frequencies $\omega_{x, y}$ & $2 \pi \times 1.0 \mathrm{MHz}$ & $2 \pi \times 0.25 \mathrm{MHz}$ \\
Typical longitudinal & & \\
$\quad$ secular frequency $\omega_{z}$ & & \\
\hline \hline
\end{tabular}




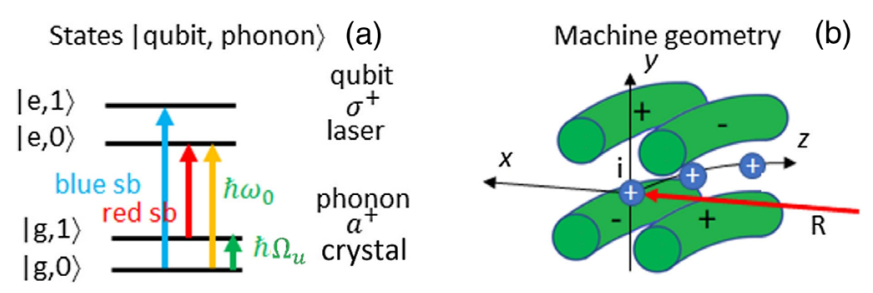

FIG. 1. (a) Simplified diagram of the valence electron state in the ion and indication of the energy levels. The qubit transition (yellow arrow) is between the ground state $|g, 0\rangle$ and the excited state $|\mathrm{e}, 0\rangle$, while the sideband transitions are shown with red and blue arrows. (b) The machine geometry with the ion chain orbiting the ring.

ions form a linear chain. 2D- and 3D ion crystals may also be formed in storage rings [33], but due to the very complicated motion of the ions in these higher dimensional systems they are not easily adaptable for quantum information processing.

To illustrate the comparison of the two concepts (a linear ion trap and a storage ring), we write the Hamiltonian governing the state and the dynamics of ions in a crystal revolving in a circular storage ring as a sum of several terms. These terms describe the internal (qubit) state of ions as a two-level system $\left(H_{I}\right)$, the dynamics of the particles moving around a circle in bending and focusing fields and interacting with each other via Coulomb forces $\left(H_{T C}\right)$, and, lastly, the atom-laser interaction Hamiltonian for individual ions $\left(H_{L}\right)$. In our brief discussion below, we will point to the formation of phonon modes as a result of the Coulomb interaction of strongly cooled ions.

The internal state of individual qubits is that of a twolevel system and is equivalent to the state of a spin- $1 / 2$ system, with the Hamiltonian given by

$$
H_{I}=\frac{1}{2} \sigma_{3} \hbar \omega_{0}+\text { H.c., }
$$

which corresponds to the transition between the internal levels in the ion separated by energy $\hbar \omega_{0}$, with $\sigma_{3}$ being the $z$-component of the Pauli matrix responsible for changing the qubit state after absorption or emission of a photon with frequency $\omega_{0}$.

The second Hamiltonian $H_{T C}$ describes the motion of $N$ ions orbiting in a storage ring and interacting with each other via Coulomb forces. We write the Hamiltonian, following Wei and Sessler [53 $]^{1}$ and switching to the nonrelativistic approximation. The units of length are measured in characteristic distance, which gives a measure of the separation between the particles in the string, $\xi=\sqrt[3]{r_{0} \frac{c^{2}}{\omega_{\mathrm{rev}}{ }^{2}}}$, where $r_{0}=\frac{1}{4 \pi \epsilon_{0}} \frac{q^{2}}{M c^{2}}$ is the classical ion radius, with $q$ and $M$ being the ion's charge and mass, respectively,

\footnotetext{
${ }^{1}$ The Hamiltonian formalism has been also presented in [5457].
}

and $\omega_{\mathrm{rev}}=\frac{\nu}{R}$ is the revolution frequency of a reference ion, $R$ is the ring bending radius and $v$ is the reference ion velocity. The energy is measured in the units of $M v^{2} \frac{r_{0}}{\xi}$. The Hamiltonian then becomes

$$
\begin{aligned}
H_{T C}= & \sum_{j}^{N} x_{j} p_{z j}+\sum_{j}^{N}\left(\frac{p_{z j}^{2}+p_{x j}^{2}+p_{y j}^{2}}{2}+\frac{\omega_{x}^{2} x_{j}^{2}+\omega_{y}^{2} y_{j}^{2}}{2}\right) \\
& +\sum_{j \neq i}^{N-1} \frac{1}{\sqrt{\left(z_{j}-z_{i}\right)^{2}+\left(x_{j}-x_{i}\right)^{2}+\left(y_{j}-y_{i}\right)^{2}}}+U_{\mathrm{rf}},
\end{aligned}
$$

where $\left(x_{j}, y_{j}, z_{j}\right)$ and $\left(p_{x j}, p_{y j}, p_{z j}\right)$ are the coordinates and momentum components of the ion $j$, and $\omega_{x, y}$ are the transverse oscillation frequencies of the particles in the ring lattice calculated under the smooth approximation. For the purpose of this paper, we will not be including a specific form of the longitudinal potential $U_{\text {rf }}$ since there is no longitudinal confinement in the ion ring being considered.

This Hamiltonian describes the motion of $N$ particles with respect to a reference particle moving with constant momentum $p_{0}=M v$. As compared to a linear ion trap, where ions are moving around their equilibrium positions defined by the external potential and their mutual Coulomb repulsion, the moving beam of $N$ ions in the ring has the total kinetic energy of $N \frac{p_{0}^{2}}{2}$, which is not included in Eq. (2). We note that in the conventional linear ion trap $p_{0}=0$, since the ions are at rest, and the string forms a straight line $(R \equiv \infty)$ instead of a circle.

The ions in the crystal are coupled via Coulomb interaction and, at sufficiently low temperatures, communicate with each other via phonon interaction. To demonstrate the origin of phonon modes, we focus on the transverse part of the Hamiltonian (2) given by $H_{u}=\sum_{j}^{N}\left(\frac{p_{u j}^{2}}{2 M}+\frac{M \omega_{u}^{2} u_{j}{ }^{2}}{2}\right)+V(u)$, where $V(u)$ is the contribution from the Coulomb interaction between the ions. In Refs. [53-57] the authors linearized the Coulomb potential by expanding it around the equilibrium point of every ion in the moving chain. Obtained equations of motion correspond to oscillators characterized by a set of frequencies $\Omega_{u}=\left(\Omega_{x l}, \Omega_{y k}, \Omega_{z n}\right)$ that are the common modes of the crystal's motion with $l, k, n=1 \ldots N$.

Next, using the technique described in Refs. $[58,59] H_{u}$ is converted from the classical to the quantum form. We write $H_{u}=\frac{1}{2 M} \sum_{j} P_{j}^{2}+\frac{M}{2} \sum_{j} \Omega_{u j}^{2} Q_{j}{ }^{2}$ with canonical momentum $P_{j}$ and it's conjugate $Q_{j}$ and introduce operators,

$$
\begin{aligned}
P_{j} \rightarrow \hat{P}_{u j} & =i \sqrt{\frac{\hbar M \Omega_{u j}}{2}}\left(a_{j}^{+}-a_{j}\right), \\
Q_{j} \rightarrow \hat{Q}_{j} & =i \sqrt{\frac{\hbar}{2 M \Omega_{u j}}}\left(a_{j}^{+}+a_{j}\right) .
\end{aligned}
$$


$H_{u}$ transforms to

$$
H_{u}=\sum_{j} \hbar \Omega_{u j}\left(a_{j}^{+} a_{j}+\frac{1}{2}\right)
$$

where $a_{j}$ and $a_{j}^{+}$are the harmonic oscillator annihilation and creation operators for the normal mode $j$, respectively, and $\hbar \Omega_{u j}$ is the quantum of energy in this mode. We note that the classical motion of the crystal with the frequencies given by $\Omega_{u j}$ is now quantized, and the interaction of ions in the string, orbiting around the ring, can be represented as the exchange of phonons via common modes of the ions' motion. We also point out that when the number of ions $N$ is large, the density of these normal modes becomes high, and spectrally resolving these modes becomes increasingly difficult.

Now, we will look at the first term in Eq. (2) that corresponds to the motion of particles around a ring with radius $R$ and describes the shear of particle orbits with respect to the momentum deviations $p_{z j}$. For a string of cold ions, considered in our paper, displacements $x_{j}$ are small and this term is negligible as the conditions for enabling quantum logic gates between the ions require the ions to be localized on a short distance scale around their equilibrium positions in the string. We will estimate this scale in Sec. IV and will confirm that the orbit deviations $\sqrt{\left\langle u_{j}^{2}\right\rangle}$, where $u=(x, y)$ is small for a cold ion beam. Therefore, in $H_{T C}$ we neglected higher order terms, proportional to $u^{n}, n>2$.

The third, laser-atom interaction Hamiltonian $H_{L}$, connects the three interactions, i.e., with the laser field, with the trap or ring fields, and with the Coulomb field of the crystal. The resulting expression is obtained by applying the rotating wave and dipole approximations and dropping the higher order terms [22],

$H_{L}=\frac{\hbar \Omega_{R}}{2} \sigma^{+} \exp \left\{-i \eta\left(a e^{-i \Omega_{u} t}+a^{+} e^{i \Omega_{u} t}\right)\right\}+$ H.c.

Here, $\Omega_{R}$ is the Rabi frequency of the transition, $\sigma^{+}$is the spin raising operator, and $\eta=\sqrt{\frac{\omega_{R}}{\omega_{u}}}$ is the Lamb-Dicke parameter, with $\omega_{R}=2 \pi^{2} \hbar c^{2} /\left(M \omega_{0}^{2}\right)$ being the recoil frequency of an ion. It is important to note that the nature of the interaction between the laser and the ions in a storage ring is essentially pulsed, since the particles are moving with constant velocity and pass through the fixed laser beam with a finite width.

Having written the principal energies of the problem, we are now equipped with the formalism to look for differences between a typical linear ion trap used in modern QC apparatus and a storage ring. To facilitate this comparison, we selected two subjects for the following discussion. We took an example of the ion trap from the
University of Washington linear ion trap experimental setup [21] and picked PALLAS [33] for the storage ring.

We emphasize the following differences between a storage ring and a linear ion trap: (i) large number of ions, (ii) ions are in motion, (iii) motion takes place around a macroscopic circular-like orbit, and (iv) ions are effectively interacting with a pulsed laser beam.

We detail these differences in the subsequent chapters of the paper and evaluate gaps between the main parameters of the ion crystals achieved in rings vs those required for QC. To conclude the paper, we outline some critical technologies that need to be established to bridge these gaps and to enable a large-scale QC in a storage ring.

\section{A MOVING ION}

In a storage ring, the ions move around a closed, circlelike orbit with a constant velocity, with an energy that ranges from $1 \mathrm{eV}$ [33] to on the order of $100 \mathrm{keV}$ [27] in mass-spectrometry setups, to a $\mathrm{TeV}$ scale in high-energy physics colliders [60]. The ions' motion around the machine orbit always comes with some velocity spread due to the finite phase space volume occupied by the beam. The velocity spread corresponds to the finite temperature of the ions' motion. Since the beam consists of many ions, it is customary to adopt a statistical beam description, with the longitudinal temperature averaged across all ions as $k_{B} T_{\|}=\frac{\left\langle p_{z}^{2}\right\rangle}{2 M}$. Spread of the particles' momenta, expressed in the second term of $H_{T C}$ in Eq. (2), defines the ions' temperature due to the difference in the energy of ions. As shown in Ref. [61], ions in the crystalline beam with a finite transverse width form a pattern or a spread of velocities in the corotating beam frame. This velocity spread corresponds to a temperature $T_{\|}$of the ions' motion, which is proportional to $1 / Q_{x}^{2}$, where $Q_{x}$ is the horizontal tune of the machine.

Another limitation of the ions' temperature, this time the transverse one, $T_{\perp}$, comes from the finite density of the focusing elements in the ring and the strength of the ring optics. Particles oscillate between the focusing lenses in the ring, which leads to a spread of the transverse velocities. The corresponding temperature [61] is proportional to the square of the betatron phase advance per cell $Q_{x} /$ Cells.

Considerations above lead to the two conclusions on limitations associated with the ions' motion. The first conclusion is that both $T_{\perp}$ and $T_{\|}$favor rings with high periodicity (or large number of cells) and strong focusing (or high value of the horizontal tune). In this regard, if we compare a synchrotron-like ring with an RFQ ring, we will see a clear advantage of the latter. Indeed, in Table 2, we compared the Aarhus STorage RIng in Denmark (ASTRID) ring based on the separate magnets [44] with the PALLAS RFQ [31].

The second conclusion is that for a realistic ring both of the apparent temperatures $T_{\perp}$ and $T_{\|}$are above the sub-mK 
TABLE II. Comparison of two types of storage rings with crystalline beams and a linear ion trap.

\begin{tabular}{lccc}
\hline \hline Device & PALLAS & ASTRID & Ion trap \\
\hline Radius between electrodes, mm & 2.5 & None & 0.5 \\
Length of ion string, mm & 361 & 40000 & $<1$ \\
Number of ions & $10^{4}$ & $2.8 \times 10^{5} \ldots 2.8 \times 10^{8}$ & $<100$ \\
Ions' energy of motion, eV & 1 & $10^{5}$ & 0 \\
Distance between ions, um & 20 & $15-140$ & $<10$ \\
Longitudinal secular frequency $\omega_{z}$ & None & None & $2 \pi \times 200 \mathrm{kHz}$ \\
Transverse secular frequencies $\omega_{x, y}$ & $2 \pi 110 \mathrm{kHz}$ & $2 \pi \times 50 \mathrm{kHz}$ & $>2 \pi \times 1000 \mathrm{kHz}$ \\
Horizontal tune, $Q_{x}$ & 50 & 2.3 & None \\
Periodicity, $P$ & 800 & 4 & None \\
\hline \hline
\end{tabular}

range, required for performing quantum logic gates between the ions. For the case of ions in PALLAS, the reported values of longitudinal and transverse temperatures are $3 \mathrm{mK}$ and about $<0.4 \mathrm{~K}$, respectively [33], which are substantially higher than the values required for quantum computation employing transverse phonon modes. It should be noted that only longitudinal laser cooling was implemented at PALLAS, which resulted in $T_{\|}$being much lower than $T_{\perp}$.

The gap between these inherent temperatures of the ring crystals achieved to date and the levels of temperature related to the kinetic energy in a given motion mode of the modern QCs on the ion traps, draws strict requirements for the level of cooling, required to enable a $\mathrm{QC}$, based on a storage ring. There is indeed a large difference in the temperatures of the ion crystals achieved at PALLAS compared to that in the ion traps, which leads to the need for $R \& D$ focused on enabling much colder ion beams in rings dedicated to $\mathrm{QC}$ experiments.

To illustrate this gap between the noise levels in the linear ion traps used for QC and those achieved in the storage rings so far, we estimate the temperature a single ion in a trap. The residual energy of a cold ion is a sum of thermal contribution, which we will measure in phonon energies as $\left(N_{p h}+\frac{1}{2}\right) \hbar \Omega_{u}$ and the micromotion contribution, which we will neglect in comparison with the former for an ion trap. To estimate the equivalent number of phonons in the thermal component, we calculate the LambDicke parameter, taking parameters of the both ions from Table 1. For the photon transitions near $0.792 \mu \mathrm{m}$ (calcium ion) or $1.76 \mu \mathrm{m}$ (barium ion) and the phonon frequencies of $\Omega_{u} \sim 2 \pi \times 2.5$ (calcium) or $2 \pi \times 1.0 \mathrm{MHz}$ (barium), we obtain $\eta \approx 0.344$ (calcium) and 0.132 (barium) for the Lamb-Dicke parameter. In order to be well into the LambDicke regime [11,23], the number of phonons in the normal mode must satisfy $N_{p h} \ll 0.5 \eta^{-2} \approx 4$ phonons for calcium ion or 28 phonons for barium ion, which corresponds to the threshold temperature $T_{L D}$ of $0.48 \mathrm{mK}(\mathrm{Ca})$ or $1.3 \mathrm{mK}$ (Ba). In contrast, in the PALLAS ring the temperature of the vertical motion of the ${ }^{24} \mathrm{Mg}^{+}$ions was estimated to be $0.4 \mathrm{~K}$ based on the beam profile measurements (limited by the resolution of the measurement setup), which indicates the factor of 1000 of the reported beam temperature in the rings relative to the regime required for realizing the LambDicke regime. We should emphasize that we estimated the temperature for the case of $N_{p h}=0.5 \eta^{-2}$, which marks the borderline for this regime and, in reality, quantum computers operate at substantially lower temperature of the residual ion motion.

We can make a rough estimate on the spatial extent of localization of ions in a trap. Taking the ion temperature $T=1 \mathrm{mK}$, which would be just below $T_{L D}$ for the barium ion, we estimate the scale of localization of that ion as $\sqrt{y_{j}^{2}} \approx \sqrt{\frac{2 k_{B} T}{M \Omega_{y}^{2}}} \approx 50 \mathrm{~nm}$, where $k_{B}$ is the Boltzmann constant. In contrast, the rms vertical beam width of the coldest crystalline beam in PALLAS was reported as $7 \mu \mathrm{m}$ [31], which leads to the difference of three orders of magnitude between the widths of the ion strings achieved in the ion traps used for QC and the crystalline beams in the state-ofthe-art storage rings.

The required degree of temperature and the related localization of the ion's motion constitute a tough constraint on the design of a storage ring system, which, due to the desired high capacity of qubits should be quite large as compared with a modern ion trap for quantum computations with 100 ions. Now we will focus on the implications of the macroscopic dimensions of the ring orbit, which, in the case of PALLAS is $36 \mathrm{~cm}$ long, whereas, in a typical ion trap, the particle crystal is $10-100$ micron long with the ions localized on a microscopic length scales of the order of $10 \mathrm{~nm}$. There are two effects that arise from the fact that the orbit may not be perfectly aligned with the rf null (the ideal orbit defined by the centers of successive RFQs) on a macroscopic scale: the excess micromotion [62] and the ac Stark and Zeeman shifts caused by the oscillating electric and magnetic fields.

Micromotion is the driven motion of the charged particles in the rf trap at the frequency of the applied rf. 
Some degree of micromotion is unavoidable and, as long as its amplitude is small enough that the resulting frequency modulation of the laser light used for the qubit logic gates is below $10 \%$, its effect on the quantum gates is negligible. However, any excess micromotion must be minimized to meet this limit.

Excess micromotion occurs when the trapped ions' equilibrium position is shifted away from the rf null by stray electric fields. In a typical linear rf trap setup, where the ion crystal is localized to a small region near the trap center, the stray electric field is uniform (to the first order). To compensate for such a field and to bring the ions' equilibrium position close to the rf null, static offset voltages may be applied to the rf electrodes of the trap, or two additional dedicated dc electrodes may be introduced. In a storage ring, however, the ion crystal occupies an extended orbit, and the stray electric field changes around the orbit. Thus, to compensate for the excess micromotion in a storage ring, a distributed network of compensation electrodes for orbit correction will be required.

Similarly, a distributed system of the beam diagnostics devices will have to be in place to detect the crystal beam orbit in multiple locations around the ring, so that it can be corrected. In the ion storage rings, the crystalline beam diagnostics are realized either with Schottky monitors or using detection of resonant fluorescence. For the lowcurrent beams forming crystalline structures, the resolution limits for Schottky pick-ups become an obstacle; therefore, one needs to focus on realizing by a few laser-ion interaction regions where the detectors will be registering the flux of fluorescence photons. Optical detection systems, capable of measuring an ion crystal length, width, momentum spread, and spectra have been developed at experimental storage ring in Darmstadt ESR, demonstrating good accuracy [63]. Recent progress with development of broadband diode lasers and short-pulse sources motivates future $R \& D$ on a compact and highly accurate setup for diagnostics of ion crystals around macroscopic ring orbit. High sensitivity and accuracy in transverse planes are required and, together with the ability of measuring beam size and length, will enable the feedback needed for reaching stable phonon modes in the orbiting ion crystals.

A stray electric field $E_{D C}=10 \mathrm{~V} / \mathrm{m}$ applied perpendicular to the ion orbit in the storage ring trap with the radial secular frequency $\omega_{x}=2 \pi \times(200 \mathrm{kHz})$ would shift the equilibrium position of a ${ }^{40} \mathrm{Ca}^{+}\left({ }^{138} \mathrm{Ba}^{+}\right)$ion by $\Delta x \simeq e E_{D C} /\left(m \omega_{x}^{2}\right) \simeq 15 \mu \mathrm{m}(4.5 \mu \mathrm{m})$ [62]. The resulting coherent motion with an amplitude of approximately $0.45 \mu \mathrm{m} \quad(0.13 \mu \mathrm{m})$ (assuming the $\mathrm{rf}$ frequency of $10 \mathrm{MHz}$ ) would carry $16 \mathrm{meV}(4.6 \mathrm{meV})$ of average kinetic energy, with the equivalent temperature of about $190 \mathrm{mK}(55 \mathrm{mK})$. We note, however, that, since the micromotion is driven and not random, this effective temperature does not directly lead to the reduction of the quantum gate fidelity.
The extended orbit of the ions in a storage-ring trap presents a unique challenge to the micromotion compensation. In a typical linear rf trap setup, the ions occupy a very small, essentially $1 \mathrm{D}$ region of space on the order of $100 \mu \mathrm{m}$ long. Any stray dc electric field can be assumed uniform in this case, and a pair of compensation electrodes can be used to bias the trap and minimize the excess micromotion. In the storage-ring trap, the stray electric field will vary along the orbit, and will have to be compensated by a distributed network of electrodes. In a chip-scale ring trap [64], such dc bias electrodes are located every $15 \mathrm{deg}$ along the circular orbit. However, we note that precise micromotion compensation (to under $1 \mu \mathrm{m}$ ) is only of essence at the location(s) of the laser beams that drive the quantum gates, while in the remaining part of the orbit only a coarse compensation (on the order of $10 \mathrm{~s}$ of microns) would be sufficient. Designing an appropriate compensation infrastructure is another important R\&D goal.

The second effect is that of the focusing fields in an RFQ ring on the internal state of an ion when the orbit is somewhat misaligned with respect to the center of the trap. Using the expressions for transverse electric and longitudinal magnetic fields in an RFQ ring [42] and, taking the misalignment of $10 \mu \mathrm{m}$, we obtain that the orbit will sample the oscillating magnetic field of $\sim 20$ Gs amplitude and oscillating electric field of $300 \mathrm{~V} / \mathrm{m}$ amplitude. Both fields oscillate at an $\mathrm{rf}$ frequency of $10-100 \mathrm{MHz}$. These oscillating magnetic and electric fields may cause ac Zeeman and ac Stark effects, respectively, which shift the electronic energy levels of the ions. While the value of the orbit shift will vary along the orbit, each ion will follow the same path (on average) and the relevant figure is the average value of the ac Zeeman and ac Stark shift. For the optical qubits with transition frequencies in the $10^{14} \mathrm{~Hz}$ range, these fields oscillating at $10-100 \mathrm{MHz}$ would cause negligible effects, since the magnitude of the shift is inversely proportional to the detuning of the oscillating field from the atomic transition. However, if hyperfine qubits with $\sim 10 \mathrm{GHz}$ transition frequencies were to be used, the ac Zeeman effect may become noticeable and will need to be taken into account for correction of the qubit transition frequency. We note, however, that is the orbit is stable overtime, or varies slowly, then these shifts are constant or nearly constant, and only periodic recalibration of the qubits transition frequency is necessary, similar to the periodic calibration of all qubits in current QC systems [65].

\section{MOVING ION CRYSTAL}

Analysis of the Hamiltonian $H_{T C}$ in Eq. (2) leads to the discovery of phonon modes for the chain of the stored ions (3). Each ion adds three normal modes, so with $10^{5}$ ions in the crystal, there are $3 \times 10^{5}$ normal modes, each with a different frequency. The frequencies lie in a relatively 
narrow range, whose width is of the order $1 \mathrm{MHz}$, and with so many ions will form an energy band.

As the phonon modes are $3 \mathrm{D}$, in a conventional ion trap one could use either longitudinal or transverse ones [66] for creating entanglement between the ions, grouping them into a string of qubits for quantum computation. As discussed earlier [see Sec. III, the first term in the Hamiltonian $H_{T C}$, Eq. (2)], in the ringlike geometry, the horizontal and longitudinal modes are coupled, which complicates potential design of a quantum gate. In addition, the vertical modes are weakly coupled to the horizontal modes via skew error fields in the ring, however, similar to the orbit correction discussed above, one may consider a coupling correction via a system of distributed electrodes.

On the other hand, strong cooling of the moving ion strings, discussed above, is a key prerequisite to realizing the QC due to constraints imposed by design of quantum gates. Operations of the latter are based on the requirement that ions stay near the motional ground state. In the case of the fast-moving ions, their transport inherently creates a motional excitation of their state. The challenge is to develop transport protocols that minimize energy transfer between each ion's motion and the phonon coupling between ions. It has been demonstrated [46-49] that even fast, nonadiabatic shuttling operations may preserve the final state of the ions close to the motional ground state. It has also been shown that quantum information stored in both the motional and the spin degree of freedom is preserved during the process of shuttling.

In particular, in experiments [48], a single ${ }^{9} \mathrm{Be}^{+}$ion was transported by $370 \mu \mathrm{m}$ in $8 \mu \mathrm{s}$, with an energy gain of only 0.1 motional quanta. Similar results were achieved for the transport of two ions, as well as for separating chains of up to nine ions from one to two different potential wells. Separating two ions was accomplished in $55 \mu \mathrm{s}$, with excitations of about two motional quanta for each ion.

These encouraging results are still very far from the specifications necessary to enable a quantum computer with moving ions in a storage ring. Indeed, preserving the integrity of the phonon modes at the level of single motional quantum appears to be a tough goal, when one considers a meter-long orbit circumference where the particles are affected by dc and ac fields. Attaining such low-heating levels in large-scale machines is one of the R\&D efforts needed to demonstrate the suitability of the rings for quantum computations.

\section{MOVING ION CRYSTAL INTERACTING WITH A LASER}

Let us consider a simple model of the Doppler cooling. To efficiently Doppler-cool the trapped ion crystal, the reddetuned laser beams must have significant wave vector components along all three principal axes of the ion trap. In a storage ring, there is an additional consideration of the lack of the ion confinement along the orbit. If not accounted for, this would lead to the ion crystal being accelerated by the radiation pressure of the Doppler cooling laser. This effect, however, presents a unique opportunity for the ion crystal rotational velocity control [35].

Let us consider the Doppler cooling laser arrangement shown in Fig. 2. The two counter-propagating beams are both red-detuned from the atomic resonance. If the detunings are identical, and both beams have the same intensity, then the radiation pressure from both beams on the ions are equal and the ions will be stationary. However, if the frequencies of the two laser beams differ by amount $\Delta \omega$, then the ions will move in the direction of the beam that has a smaller lab frame detuning until, in the rest frame of the ions, both lasers have the same detuning due to the Doppler effect. This condition is satisfied when the velocity of the ions is given by $k v=\Delta \omega / 2$. For example, if the desired $\mathrm{Ca}^{+}$ion velocity is $100 \mathrm{~m} / \mathrm{s}$, then the detuning between the two $397 \mathrm{~nm}$ Doppler cooling lasers needs to be approximately $80 \mathrm{MHz}$.

The counterpropagating Doppler cooling beam arrangement does not, however, efficiently cool the transverse motion of the ions. To address this problem, a pair of counterpropagating laser beams may be used, also schematically shown in Fig. 2, that is perpendicular to the orbit and has substantial $k$-vector components along both transverse axes. For more efficient interaction with the ions, these laser beams' cross section may be made elliptical, extended along the orbit, using cylindrical lenses. Multiple laser cooling sections may be implemented along the orbit to lower the ion temperature even further.

The transverse temperature of the ions can be deduced from the width of the ion beam image with the resolution limited by the diffraction effects from the imaging optics [67]. This method works well when the transverse temperature is relatively high, such that the resulting spatial extent of the ion localization is larger than or comparable to the diffraction limit of the optics. For lower temperatures, in the $1 \mathrm{mK}$ regime and below, spectra data may be used both from the ion fluorescence and the absorption of the laser light. These techniques include detection of the Doppler broadening of the resonance fluorescence spectra [68], and asymmetry of the secular motion sidebands due to the ion's motion [69].

Doppler cooling may bring the ion temperature close to the Lamb-Dicke regime. Further cooling is needed, however, to achieve high-fidelity qubit gates. Since we propose to use the transverse phonons to carry quantum information, as discussed in Sec. VII, the transverse motion would have to be further cooled [13]. Vertical degree of freedom ( $y$ axis) is completely decoupled from the $x$ and $z$ motion, and can be cooled well into the Lamb-Dicke regime with $N_{p h} \ll 0.5 \eta^{-2}$ using either the Sisyphus cooling [25] or the EIT cooling [26] by appropriately tuning the same laser beams that are used for the transverse Doppler cooling. 


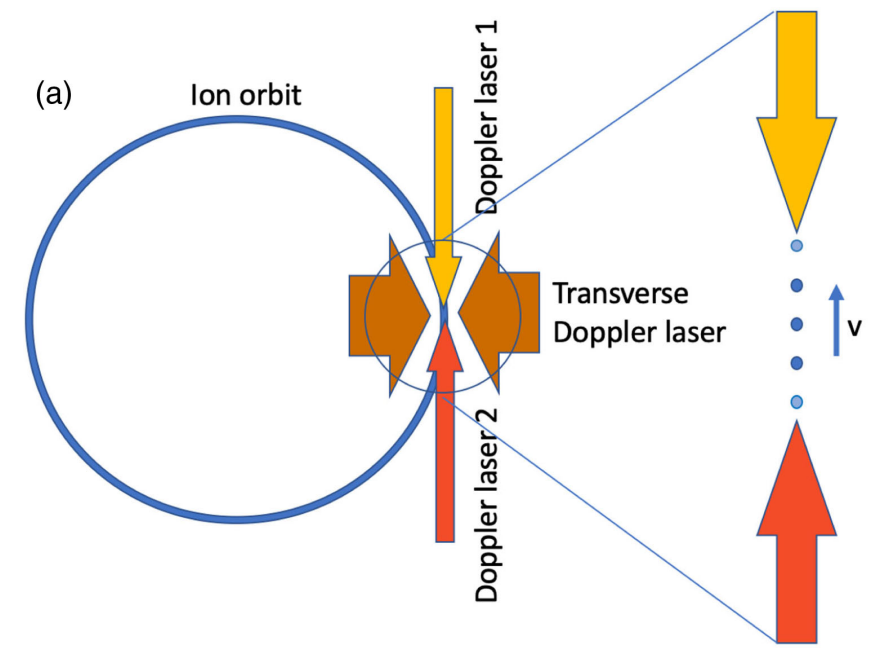

(b)

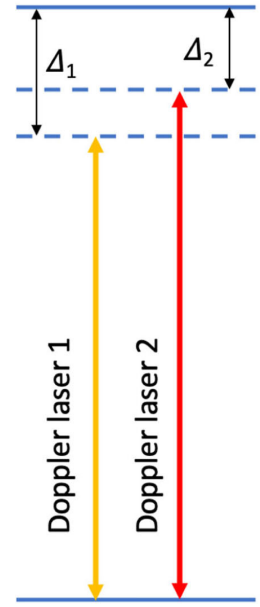

Lab frame

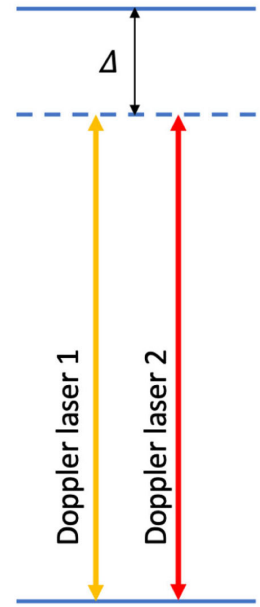

Ion frame

FIG. 2. Doppler laser cooling beam arrangement. (a) Two focused, counter propagating laser beams ("Doppler laser 1" and "Doppler laser 2") with different frequencies but equal intensities are applied tangentially to the ion orbit to cool the ion motion in the $z$-direction, as well as to set the orbital velocity. An additional pair of counterpropagating laser beams ("Transverse Doppler laser"), shaped to have an elongated profile along the ion orbit direction, is applied perpendicular to the orbit to enable cooling of the $\mathrm{x}$ and $\mathrm{y}$ directions. (b) The relevant atomic energy levels and laser detunings in the lab frame (left) and the moving Ion frame (right). In the lab frame, the detuning $\Delta_{1}$ of the "Doppler laser 1" is greater than the detuning $\Delta_{2}$ of the "Doppler laser 2." This creates a larger scattering force by the "Doppler laser 2," so that the ions are forced to move in the direction of that laser until their velocity is such that the detunings of both lasers are the same $(\Delta)$ in the Ion frame. Then the scattering forces due to both lasers are equal, and the ions move with a constant speed.

\section{QUANTUM GATES}

Now, we look at the implications of the laser interacting with ions as described by $\mathrm{H}_{L}$ in Eq. (4). Quantum logic gates on trapped ions are typically driven by the laser beams whose intensity, frequency, and phase is controlled by an rf signal generator via an AOM. After passing through the AOM, the laser beams are focused onto the ions in the trap. Following this model, we consider a laser beam, interacting with the chain of ions, which are moving through the interaction region, as opposed to being static in a conventional ion trap. We assume that the crystal orbits around the ring at $v=2.8 \mathrm{~km} / \mathrm{s}(1 \mathrm{eV}$ of kinetic energy for $\left.{ }^{24} \mathrm{Mg}^{+}\right)$as it was actually observed in the PALLAS RFQ ring. A slower beam would relax the requirements on the gate speeds as we will find out next.

Taking the separation of ions moving around with velocity $v$ in a chain crystalline beam as $20 \mu \mathrm{m}$, we assess the timing of their interaction with the laser. The laser beam crosses the ions' path with a waist of $w_{0}=10 \mu \mathrm{m}$, which corresponds to half of the separation between consecutive ions in the chain, so that the neighboring ions are not affected by the laser beam controlling the state of a given qubit. This gives the Rayleigh range of the laser beam $z_{R}=\frac{\pi w_{0}^{2}}{\lambda_{1}} \approx 0.8 \mathrm{~mm}$. The laser beam envelope as a function of the distance transverse to the orbit and the chain of ions (blue circles), together with the laser intensity distribution at the focus of the laser beam, are shown in Fig. 3.
The laser source should be turned on during the time of flight of an ion through the interaction region and then the light should be turned off so as not to affect the next ion in the chain. Turning the laser on and off with a highbandwidth $\mathrm{AOM}$ can be done with $\sim 2 \mathrm{~ns}$ rise or fall times [52]. The pulse length is then $\tau_{l}=\frac{w_{0}}{2 v} \approx 4.6 \mathrm{~ns}$, where a

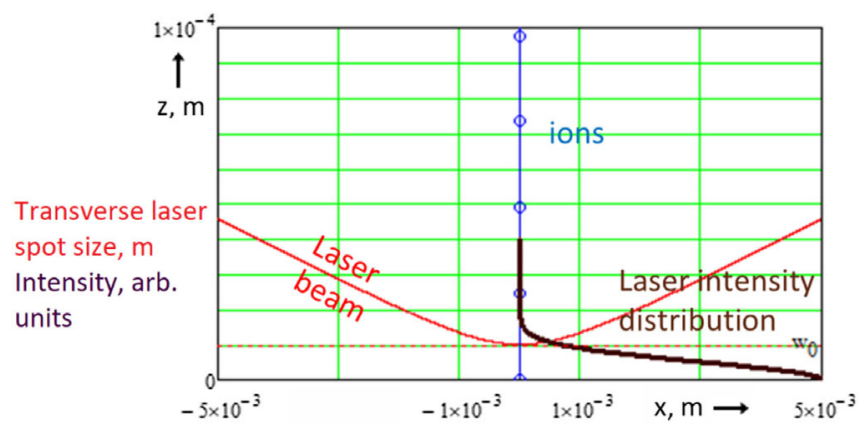

FIG. 3. Model of the quantum gate, illustrating the laser Gaussian beam transverse envelope in red, intensity distribution of the laser beam at the waist in brown, and positions of ions in the crystalline beam (blue), which is moving from bottom to top and traversing the laser beam in the interaction region. The laser waist is a half of the distance between the consecutive ions and the laser pulse length is a half of the time of flight of the ions through the same region. A single laser pulse interacts with a single ion setting the qubit's state prior to execution of a cycle of quantum computation. 
factor of 2 is taken to accommodate the ramp-up of intensity along the pulse. This is significantly shorter than the typical trapped ion quantum gate times (approximately $1 \mu \mathrm{s}$ for single-qubit gates and $100 \mu$ s for two-qubit gates when CW laser beams are used). We note that quantum gates as fast as $3 \mathrm{~ns}$ have been reported using mode-locked lasers [70].

The laser intensity necessary to drive the gates in this limited amount of time is defined by the Rabi oscillation frequency for the qubit transition. For example, a bit-flip operation on a single qubit is equivalent to a Rabi $\mu$-pulse, such that the probability of the qubit transition from state $|g, 0\rangle$ to state $|e, 0\rangle$ (and vice versa) is $P=$ $\sin \left(\Omega_{R} \tau_{l} / 2\right)^{2}=1$. For the qubit transition in $\mathrm{Ca}^{+}$at $792 \mathrm{~nm}\left(\mathrm{Ba}^{+}\right.$at $\left.1762 \mathrm{~nm}\right)$, which is an electric quadrupole transition, the Rabi frequency at the laser intensity of $\sim 5000 \mathrm{~W} / \mathrm{mm}^{2}\left(250 \mathrm{~mW} / \mathrm{mm}^{2}\right)$ was measured to be approximately $2 \pi \times 1000 \mathrm{kHz} \quad(2 \pi \times 43 \mathrm{kHz}) \quad[22,71]$, which gives a $\pi$-pulse time of about $0.5 \mu \mathrm{s}(11.6 \mu \mathrm{s})$. For an electric quadrupole transition, the Rabi frequency scales linearly with the laser intensity; thus, to achieve a $4.6 \mathrm{~ns} \pi$ pulse time, a laser intensity of about $540000 \mathrm{~W} / \mathrm{mm}^{2}$ would be needed for $\mathrm{Ca}^{+}$(about $600 \mathrm{~W} / \mathrm{mm}^{2}$ would be needed for $\mathrm{Ba}^{+}$). This is a manageable level of intensity for barium and can be realized with a $100 \mathrm{~mW}$ CW laser focused to a $10 \mu \mathrm{m}$ waist. The requirement for $\mathrm{Ca}^{+}$is much more severe. It may be possible to enable step-wise gates executed over many cycles of rotation that would relax these power requirements.

For two-qubit entangling gates, the standard protocol in the trapped ion QC is to use the quantized collective motion of all ions in the crystal as the quantum "data bus" to couple the individual qubits [72,73]. Such gates have to be performed by adiabatically exciting the quantized ion motion using lasers that couple the qubit spin and motional states, as in the Hamiltonian presented in Eq. (4), in the resolved sideband regime. However, with $\sim 10^{5}$ normal modes achieving this regime becomes impractical. For such a large and distributed set of qubits, the all-to-all qubit connectivity, which is enabled by the resolved sideband regime in a linear ion trap, cannot be realized, and different types of two-qubit and multiqubit gates will have to be developed that involve only the nearest neighbor qubits [74-76]. Optical power requirement for these gates to be implemented on the ns time scales is likely to significantly exceed the power available from the existing $\mathrm{CW}$ laser sources. Development of the novel protocols that involve ultrafast pulsed lasers is another direction for the R\&D. We note a recently proposed "linear-tape" architecture of a trapped-ion quantum computer [77], which bears some relevance to the storage-ring architecture and may serve as a useful starting point for future development. Development and implementation of new types of robust quantum gates in large ensembles of trapped ions is an active area of theoretical and experimental research [66,76-80].
To realize such fast quantum gates, an adequate AOM system that is capable of addressing every ion in the crystal is necessary. This will require the AOM to switch at the rate of $140 \mathrm{MHz}$ while generating short pulses with $\sim 2 \mathrm{~ns}$ rise or fall times and tunable intensity and polarization. Such fast modulators are available commercially (for example, the GPM-1600-400 AOM by Brimrose [52]) but may not be able to handle the amount of optical power needed. We thus conclude that future quantum computers on crystalline beams will rely on broadband modulators that will be capable of handling high-optical power while generating short pulses with high rf bandwidth in the range of 100s of $\mathrm{MHz}$. This is another important direction for future $\mathrm{R} \& \mathrm{D}$ focusing on realizing QC with moving ions.

One other important conclusion is that the ions' velocity plays a pivotal role in every aspect of the pulsed and time-offlight techniques that will be used in quantum computers. The minimal sustainable value of the ion's velocity is limited by the balance of ion heating by various environmental factors and field errors, and the efficiency of cooling being localized to a fraction of the ring circumference. Reducing the ions' velocity in storage rings while preserving the strong focusing of ion orbits and a high degree of cooling is another formidable task for future $R \& D$.

\section{QUANTUM GATE WITH A LARGE NUMBER OF IONS}

In addition to the laser cooling and orbit control problems, the very large number of ions in the storagering quantum computer presents other unique challenges relevant to QC operations: (i) keeping track of the individual qubits; (ii) identifying and mitigating qubit loss due to collisions with the residual background gas; and (iii) detecting ion reordering due to such collisions.

To address (i), we propose a stochastic method for keeping track of the individual ion qubits. When loading the ring, another ion species is randomly mixed with the main qubit ion in a small proportion (1:10 ratio or so). The other species may be simply a different isotope of the same species as the main qubit, such as ${ }^{43} \mathrm{Ca}$ if the main qubit ion is ${ }^{40} \mathrm{Ca}$ or ${ }^{136} \mathrm{Ba}$ if the main qubit is ${ }^{138} \mathrm{Ba}$. Then, when laser cooled, the admixture ions will not fluoresce and will present themselves as dark gaps in the chain of bright qubit ions. These bright/dark patterns will be random and unique at every point along the linear ion crystal, thus allowing one to identify the qubit ions. Figure 4 shows an example of a small trapped barium ion chain with the ${ }^{138} \mathrm{Ba}$ ions being bright and ions of a different isotope $\left({ }^{136} \mathrm{Ba}\right)$ being dark.

For issues (ii) and (iii), we note that if a mismatch in the bright or dark pattern is detected, it may be due to ion loss or ion reordering as a result of background gas collisions. If such an event is detected, then the subset of qubits involved in it must be reset and reencoded. Development of 

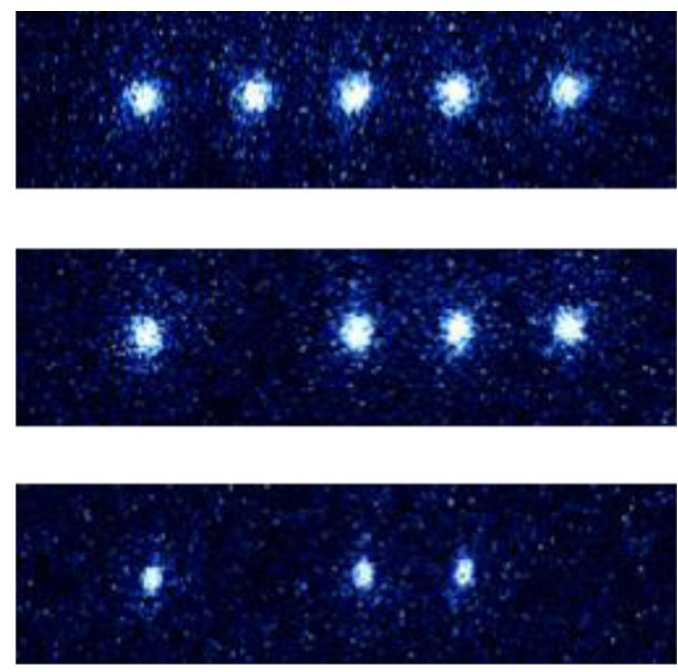

FIG. 4. Intensified charge-coupled device CCD camera images of trapped Ba ions in a University of Washington linear trap. Only the ${ }^{138} \mathrm{Ba}$ ions are resonant with the Doppler cooling laser, so only they are visible. ${ }^{136} \mathrm{Ba}$ ions appear as dark gaps in the chain. Top: A five-ion chain composed of five ${ }^{138} \mathrm{Ba}$ ions. Middle: A five-ion chain composed of four ${ }^{138} \mathrm{Ba}$ ions and one ${ }^{136} \mathrm{Ba}$ ion (second from the left). Bottom: A five-ion chain composed of three ${ }^{138} \mathrm{Ba}$ ions and two ${ }^{136} \mathrm{Ba}$ ions (second and fifth from the left).

fault-tolerant protocols that allow mitigating such problems is another direction for future $R \& D$.

The "switching rate" of an ordinary trapped ion quantum processor is limited by the minimum duration of the laser pulses interacting with the ions necessary to complete a quantum gate. According to Steane [11], an estimate for the maximum Rabi frequency to drive motional sidebands without affecting the nearby quantum motional states in a crystal with $\mathrm{N}$ ions is given by $\Omega_{N}=\eta \Omega_{R} / \sqrt{N}$, where the Rabi frequency $\Omega_{R}$ corresponds to a single ion transition. Then the switching rate is thus set by the inverse of the Rabi $2 \pi$-pulse time, the Lamb-Dicke parameter, and the total ion number.

Two problems arise here when considering a storage-ring QC architecture. The first is related to the duration of the pulse, limited by the time of flight of the ion through the ionlaser interaction zone, as described in Sec. VII. The other issue is the scaling of the switching rate with the number of ions at large values of N. Indeed, taking the single-ion Rabi frequency of $2 \pi \times 218 \mathrm{MHz}$, as required for a single-qubit gate to be completed in $4.6 \mathrm{~ns}$, we estimate the two-qubit gate time for 100 ions as $230 \mathrm{~ns}$ (taking $\eta=0.2$ ). For $10^{5}$ ions, the two-qubit gate time becomes $7.3 \mu \mathrm{s}$, which would severely limit the computing rate if traditional trapped ion QC architectures were used.

Another, more severe limitation on the gate speed comes from the fact that the phonon spectrum of a crystal of $10^{5}$ ions is very dense. If the transverse center of mass frequency is of order $1 \mathrm{MHz}$, then the $10^{5}$ normal modes lie in a band of approximately $1 \mathrm{MHz}$ in widths, thus leaving approximately $10 \mathrm{~Hz}$ per mode. Thus, in order to achieve the resolved sideband regime, the operations must be performed slower than $0.1 \mathrm{~s}$. We conclude that the regular approach used in the ion traps for performing quantum gates is not suitable for the storage ring $\mathrm{QC}$, and fast quantum gates will need to be developed that do not require a single normal mode addressing and work on small, local subsets of qubits [53-55]. Piecewise gate implementation may also be of interest here, as mentioned above, where the entire gate is split into a series of smaller steps, each completed during one passage of the ions through the interaction region.

\section{SUMMARY}

In this paper, we analyzed a new, interesting conceptturning storage rings with crystalline beams into a giant trap for QC. It is clear that there are gaps between the quality of crystalline beams achieved to date and what is required to enable quantum computations. The motion of particles along a macroscopic orbit, the high-thermal temperature of the ions' motion, the limited time for interaction with cooling and gate lasers, sensing and correcting the ions' motionthese, and other challenges present clear objectives for future R\&D.

Below we focus on each of the six R\&D objectives that we outlined in this paper. Following the flow of our logic above, we move from a single ion to the moving ion crystal and, then, to that with a large number of ions, interacting with lasers.

The first objective is to produce much colder beams than those available today and learn how to sustain their low temperature in steady state. The goal of reducing the ion temperature by an order of magnitude, relative to the range demonstrated at PALLAS, would be the first significant step toward producing beam quality suitable for enabling quantum computations. Proof-of-principle experiment, based on the Doppler cooling followed by the EIT cooling 26]], is conceivable to, first, model and, then, design for a specialized storage ring.

The second objective is to advance engineering of storage rings to reduce the amount of thermal and rf noise. An interesting experiment could be to include a special straight section into an existing crystalline ion ring, such as integrable optics Test accelerator [81] at Fermilab, with broadly variable temperature and, using narrowband laser as a "thermometer," study the ion beam heating rates and their lowest achievable temperatures.

The third objective is to develop a compact and highly accurate diagnostics and control systems of the ion crystals' orbit around the macroscopic ring. Design of a singleparticle beam position monitor, either photon or rf, with the goal of demonstrating both the turn-by-turn detection and slow acquisition, of the single particle and crystalline beam position with an accuracy of $10 \mu \mathrm{m}$ appears to be an exciting goal for future engineering of QC beam 
diagnostics. We foresee using high-fidelity detection of the laser-induced fluorescence for the photon BPM and cryogenically cooled electrode setup for the rf BPM similar to those used in penning traps [82]. Single-photon sensitive complementary metal-oxide-semiconductor cameras [83] with quantum efficiency of $\sim 20 \%$ can serve as photon detectors for the expected single photon flux of $10^{6} / \mathrm{s}$ per ion. The rf BPMs, if feasible, can also be purposed for the orbit correction system by integrating dc-offsets to the sensing electrodes.

Interaction with lasers present a number of specific and principal challenges. The fourth objective is to develop novel protocols for twoqubit and multiqubit gates that involve ultrafast pulsed lasers. The goal here is to build theoretical models for the basic quantum logic operations suitable for the large, moving ion crystals. Experimental demonstrations of the feasibility of such quantum gates may be performed in the regular linear ion traps where the motion of the ion crystal can be induced by modulating the endcap electrode voltages.

Additionally, protecting the quantum states of qubits from decoherence and loss of quantum states in a storage ring setup requires development of specific fault-tolerant protocols. While standard QEC protocols used in ion traps may be applicable to combat the decoherence, there are special considerations for large moving ion crystals.

As we have shown above, the ultrafast lasers appear to be necessary to enable these quantum logic gates. The fifth objective is to design and build a broadband modulator that is capable of handling high-optical power of such lasers while generating short pulses with high-rf bandwidth in the range of 100s of MHz. A multichannel AOM producing $2 \mathrm{~ns}$ rise and fall time pulses at a gigahertz rate, while sustaining Watts of optical power with intensities of order $\mathrm{MW} / \mathrm{mm}^{2}$ stands as a goal for realization of the quantum logic gates in the moving ion crystals.

On the other hand, we saw that the straightforward method of alleviating tight requirements on the laser systems and AOMs is reducing the ions' velocity in storage rings while preserving the strong focusing of ion orbits and a high degree of cooling. Addressing this, sixth, objective, we arrive at the need of developing a "thermodynamic" particle tracking code that takes into account radiation, absorption of photons, finite temperature of surrounding chambers, and vacuum conditions. The code should also be capable of computing noise in the particle beams, cooling effects, evaluating, and keeping track of the Coulomb fields during beam propagation around the ring. Equipped with such code, we will be in the position to make headway in optimizing the ring models under conditions of heating to test the use of complex cooling techniques and to understand the limit of lowest ion crystal velocities, with the minimum goal of lowering the velocity by at least a factor of ten compared to $2.8 \mathrm{~km} / \mathrm{s}$ achieved in PALLAS.
Concluding our paper and being now equipped with considerations on the scale of the problem, we may ask ourselves: how conceivable is the concept of macroscopic storage rings running $10^{5}$ ions, entangled and coupled to the rf pick-ups and lasers? At this point this question remains open, since the gaps between what has been demonstrated and what is required are so large. However, the reward for putting such a system to work is dramatic: the highly redundant and reliable operation of the fastest-ever computer with parallel processors, capable of reaching new horizons in image recognition, data mining, and solving other problems of extreme complexity. We believe that when the six objectives described above were met, we will be in the position to design an experiment and to code the very first simple quantum program in a crystalline beam of particles revolving around a macroscopic storage ring.

\section{ACKNOWLEDGMENTS}

The authors wish to thank Gennady Stupakov for useful comments. This research was supported by U.S. National Science Foundation Grant No. PHY-2011503.

[1] J. Preskill, Lecture notes for Physics 219 at Caltech, http:// theory.caltech.edu/ preskill/ph229.

[2] T. H. Johnson, S. R. Clark, and D. Jaksch, What is a quantum simulator?, Eur. Phys. J. Quantum Technol. 1, 10 (2014).

[3] F. Arute, K. Arya, R. Babbush et al., Quantum supremacy using a programmable superconducting processor, Nature (London) 574, 505 (2019).

[4] D. Jaksch, J. I. Cirac, P. Zoller, S. L. Rolston, R. Côté, and M. D. Lukin, Fast Quantum Gates for Neutral Atoms, Phys. Rev. Lett. 85, 2208 (2000).

[5] C. Monroe, D. M. Meekhof, B. E. King, W. M. Itano, and D. J. Wineland, Demonstration of a Fundamental Quantum Logic Gate Phys. Rev. Lett. 75, 4714 (1995).

[6] M. H. Devoret and R.J. Schoelkopf, Superconducting circuits for quantum information: An Outlook, Science 339, 1169 (2013).

[7] J. R. Petta, A. C. Johnson, J. M. Taylor et al., Coherent manipulation of coupled electron spins in semiconductor quantum dots, Science 309, 2180 (2005).

[8] P. Kok, W. J. Munro, K. Nemoto, T. C. Ralph, J. P. Dowling, and G.J. Milburn, Linear optical quantum computing with photonic qubits, Rev. Mod. Phys. 79, 135 (2007).

[9] J. R. Weber, W. F. Koehl, J. B. Varley, A. Janotti, B. B. Buckley, C. G. Van de Walle, and D. D. Awschalom, Quantum computing with defects, Proc. Natl. Acad. Sci. U.S.A. 107, 8513 (2010).

[10] N. Friis, O. Marty, C. Maier et al., Observation of Entangled States of a Fully Controlled 20-Qubit System, Phys. Rev. X 8, 021012 (2018).

[11] A. Steane, The ion trap quantum information processor, Appl. Phys. B 64, 623 (1997). 
[12] P. Wang, C. Y. Luan, M. Qiao, M. Um, J. Zhang, Y. Wang, X. Yuan, M. Gu, J. Zhang, and K. Kim, Single ion qubit with estimated coherence time exceeding one hour, Nat. Commun. 12233 (2021).

[13] K. Wright et al., Benchmarking an 11-qubit quantum computer, Nat. Commun. 10, 5464 (2019).

[14] V. M. Schäfer, C. J. Ballance, K. Thirumalai, L. J. Stephenson, T. G. Ballance, A. M. Steane, and D. M. Lucas, Fast quantum logic gates with trapped-ion qubits, Nature (London) 555, 75 (2018).

[15] J. E. Christensen, D. Hucul, W. C. Campbell, and W. R. Hudson, High-fidelity manipulation of a qubit enabled by a manufactured nucleus, npj Quantum Inf. 6, 35 (2020).

[16] L. A. Zhukas, P. Svihra, A. Nomerotski, and B. B. Blinov, High-fidelity simultaneous detection of trapped ion qubit register, arxiv:2006.12801.

[17] W. D. Phillips, Nobel lecture: Laser cooling and trapping of neutral atoms, Rev. Mod. Phys. 70, 721 (1998).

[18] M. Bonitz, P. Ludwig, H. Baumgartner et al., Classical and quantum Coulomb crystals, Phys. Plasmas 15, 055704 (2008).

[19] M. G. Raizen, J. M. Gilligan, J. C. Bergquist, W. M. Itano, and D. J. Wineland, Ionic crystals in a linear Paul trap, Phys. Rev. A 45, 6493 (1992).

[20] T. Ruster, C. T. Schmiegelow, H. Kaufmann, C. Warschburger, F. Schmidt-Kaler, and U. G. Poschinger, A long-lived Zeeman trapped-ion qubit, Appl. Phys. B 122, 254 (2016).

[21] M. R. Dietrich, N. Kurz, T. Noel, G. Shu, and B. B. Blinov, Hyperfine and optical barium ion qubits, Phys. Rev. A 81, 052328 (2010).

[22] R. Blatt, H. Häffner, C. F. Roos, C. Becher, and F. SchmidtKaler, Ion trap quantum computing with $\mathrm{Ca}^{+}$ions, Quantum Inf. Process. 3, 61 (2004).

[23] D. J. Wineland, Experimental issues in coherent quantumstate manipulation of trapped ions, J. Res. Natl. Inst. Stand. Technol. 103, 259 (1998).

[24] D. J. Wineland and H. Dehmelt, Proposed $10^{14} \Delta \nu<\nu$ Laser Fluorescence Spectroscopy on $\mathrm{Tl}^{+}$Mono-Ion Oscillator III, Bull. Am. Phys. Soc. 20, 637 (1975).

[25] J. Dalibard and C. Cohen-Tannoudji, Laser cooling below the Doppler limit by polarization gradients: Simple theoretical models, J. Opt. Soc. Am. B 6, 2023 (1989).

[26] R. Lechner, C. Maier, and C. Hempel, Electromagnetically-induced-transparency ground-state cooling of long ion strings, Phys. Rev. A 93, 053401 (2016).

[27] K. Blaum, High-accuracy mass spectrometry with stored ions, Phys. Rep. 425, 1 (2006).

[28] W. Paul, Electromagnetic traps for charged and neutral particles, Rev. Mod. Phys. 62, 531 (1990).

[29] W. Paul and H. Steinwedel, Ein neues Massenspektrometer ohne Magnetfeld, Phys. Inst. Univ. Bonn 8a, 448 (1953).

[30] J. Drees and W. Paul, Beschleunigung yon Elektronen in einem Plasmabetatron, Z. Phys. 180, 340 (1964).

[31] U. Schramm, T. Schatz, M. Bussmann, and D. Habs, Storage of crystalline beams, in Proceedings of 2003 PAC Conference, 0-7803-7739-9 (2003), pp. 112-116, https:// www.researchgate.net/publication/

4069747_Storage_of_crystalline_ion_beams.
[32] U. Schramm, T. Schätz, and D. Habs, Laser-cooling of ions and ion acceleration in the RF-quadrupole ring trap PALLAS, AIP Conf. Proc. 576, 667 (2001).

[33] T. Schätz, U. Schramm, and D. Habs, Crystalline ion beams, Nature (London) 412, 717 (2001).

[34] U. Schramm, T. Schätz, and D. Habs, Bunched Crystalline Ion Beams, Phys. Rev. Lett. 87, 184801 (2001).

[35] U. Schramm, T. Schätz, and D. Habs, Three-dimensional crystalline ion beams, Phys. Rev. E 66, 036501 (2002).

[36] T. Shaftan, Proposal on building quantum computer using crystalline beams of ions in a synchrotron, Technical Report No. NSLSII-ASD-TN- 299, 2019.

[37] T. Shaftan, On challenges with using crystalline beams of ions as a medium for quantum computers, Technical Report No. NSLSII-ASD-TN-309, 2019.

[38] K. A. Brown and T. Roser, Towards storage rings as quantum computers, Phys. Rev. Accel. Beams 23, 054701 (2020); K. A. Brown and T. Roser, U.S. Patent Application Serial No. 16/864, 332 (2020).

[39] U. Schramm, T. Schatz, M. Bussmann, and D. Habs, Spatial compression of bunched crystalline ion beams, Phys. Scr. T T104, 189 (2003).

[40] J. Wei, X.-P. Li, and A. Sessler, Crystalline beam ground state, BNL-52381, UC-414, 1993.

[41] J. P. Schiffer, Crystalline beams, in Proceedings of the 1996 Particle Accelerator Conference (1996), pp. 3164, https://inis.iaea.org/collection/NCLCollectionStore/ _Public/32/016/32016416.pdf.

[42] A. G. Ruggiero, Crystalline beams, in Proceddings of the 2nd ICFA Workshop on The Physics of High Brightness Beams, 1999, BNL-66946.

[43] X.-P. Li, H. Enokizono, H. Okamoto, Y. Yuri, A. M. Sessler, and J. Wei, Phonon spectrum and the maintenance condition of crystalline beams, Phys. Rev. ST Accel. Beams 9, 034201 (2006); S. Schröder et al., First laser cooling of relativistic ions in a storage ring, Phys. Rev. Lett. 64, 2901 (1990); J. S. Hangst, M. Kristensen, J. S. Nielsen, O. Poulsen, J. P. Schiffer, and P. Shi, Laser cooling of a stored ion beam to $1 \mathrm{mK}$, Phys. Rev. Lett. 67, 1238 (1991).

[44] N. Kjaergaard, Methods in laser cooling of ions in storage rings and traps, Ph.D. Thesis, Institute of Physics and Astronomy, Aarhus University, 2001.

[45] G. Pagano et al., Cryogenic trapped-ion system for large scale quantum simulation, Quantum Sci. Technol. 4, 014004 (2018).

[46] D. Kielpinski, C. Monroe, and D. J. Wineland, Architecture for a large-scale ion-trap quantum computer, Nature (London) 417, 709 (2002).

[47] A. Walther, F. Ziesel, T. Ruster, S. T. Dawkins, K. Ott, M. Hettrich, K. Singer, F. Schmidt-Kaler, and U. Poschinger, Controlling Fast Transport of Cold Trapped Ions, Phys. Rev. Lett. 109, 080501 (2012).

[48] R. Bowler, J. Gaebler, Y. Lin, T. R. Tan, D. Hanneke, J. D. Jost, J. P. Home, D. Leibfried, and D. J. Wineland, Coherent Diabatic Ion Transport and Separation in a Multizone Trap Array, Phys. Rev. Lett. 109, 080502 (2012).

[49] R. B. Blakestad, C. Ospelkaus, A. P. VanDevender, J. M. Amini, J. Britton, D. Leibfried, and D. J. Wineland, HighFidelity Transport of Trapped-Ion Qubits through an 
X-Junction Trap Array, Phys. Rev. Lett. 102, 153002 (2009).

[50] J. M. Pino, J. M. Dreiling, C. Figgatt et al., Demonstration of the QCCD trapped-ion quantum computer architecture, arXiv:2003.01293v2.

[51] C. Monroe, R. Raussendorf, A. Ruthven, K. R. Brown, P. Maunz, L.-M. Duan, and J. Kim, Large scale modular quantum computer architecture with atomic memory and photonic interconnects, Phys. Rev. A 89, 022317 (2014).

[52] Brimrose corporation of America, www.brimrose.com.

[53] J. Wei, X.-P. Li, and H. Okamoto, Crystalline beam studies with Andy Sessler, in Proceedings of the COOL2015, Newport News, VA, USA (2015), pp. 155-164, https:// accelconf.web.cern.ch/cool2015/index.html.

[54] H. Okamoto, Single-particle orbit equation in crystalline beams, Phys. Plasmas 9, 322 (2002).

[55] J. Wei, X.-P. Li, and A. Sessler, The low-energy state of circulating stored ion beams: Crystalline beams, Technical Report No. LBL-35322, UC-414, 1994.

[56] I. Meshkov, T. Katayama, A. Sidorin, A. Smirnov, E. Syresin, G. Trubnikov, and H. Tsutsui, Simulation of crystalline beams in storage rings using molecular dynamics technique, Nucl. Instrum. Methods Phys. Res., Sect. A 558, 303 (2006).

[57] A. Pisent, Theory of crystalline beams, Hyperfine Interact. 115, 3 (1998).

[58] G. Morigi and S. Fishma, The dynamics of an ion chain in a harmonic potential, Phys. Rev. E 70, 066141 (2004).

[59] D. F. V. James, Quantum dynamics of cold trapped ions with application to quantum computation, arXiv:quant-ph/ 9702053v1.

[60] https://atlas.cern/updates/atlas-feature/higgs-boson.

[61] J.P. Schiffer, The physics of crystalline beams, in Proceedings of the 31st ELOISATRON Workshop on crystalline Beams and Related Issues, Erice, Italy, 1995, https:// www.osti.gov/servlets/purl/208369.

[62] D. J. Berkeland, J. D. Miller, J. C. Bergquist, W. M. Itano, and D. J. Wineland, Minimization of ion micromotion in a Paul trap, J. Appl. Phys. 83, 5025 (1998)

[63] M. Bussmann, U. Schramm, G. Birkl et al., All-optical ion beam cooling and online diagnostics at relativistic energies, in Proceedings of the Cool 2009, Lanzhou, China, 2009, MOA1MCIO01, https://accelconf.web.cern.ch/ COOL2009/index.htm.

[64] B. Tabakov, F. Benito, M. Blain, C. R. Clark, S. Clark, R. A. Haltli, P. Maunz, J. D. Sterk, C. Tigges, and D. Stick, Assembling a ring-shaped crystal in a microfabricated surface ion trap, Phys. Rev. Applied 4, 031001 (2015).

[65] Y. Nam, J. S. Chen, N. C. Pisenti et al., Ground-state energy estimation of the water molecule on a trapped-ion quantum computer, npj Quantum Inf. 6, 33 (2020).

[66] S.-L. Zhu, C. Monroe, and L. M. Duan, Trapped Ion Quantum Computation with Transverse Phonon Modes, Phys. Rev. Lett. 97, 050505 (2006).

[67] B. B. Blinov, R. N. Kohn, Jr., M. J. Madsen, P. Maunz, D. L. Moehring, and C. Monroe, Broadband laser cooling of trapped atoms with ultrafast pulses, J. Opt. Soc. Am. B 23, 1170 (2006).

[68] D. J. Winel and W. M. Itano, Spectroscopy of a single $\mathrm{Mg}^{+}$ ion, Phys. Lett. 82A, 75 (1981).

[69] F. Diedrich, J. C. Bergquist, Wayne M. Itano, and D. J. Wineland, Laser Cooling to the Zero-Point Energy of Motion, Phys. Rev. Lett. 62, 403 (1989).

[70] J. Mizrahi, C. Senko, W. C. Campbell, C. W. S. Conover, and C. Monroe, Ultrafast Spin-Motion Entanglement and Interferometry with a Single Atom, Phys. Rev. Lett. 110, 203001 (2013).

[71] T. Noel, M. R. Dietrich, N. Kurz, G. Shu, J. Wright, and B. B. Blinov Adiabatic passage in the presence of noise, Phys. Rev. A 85, 023401 (2012).

[72] J. I. Cirac and P. Zoller, Quantum Computations with Cold Trapped Ions, Phys. Rev. Lett. 74, 4091 (1995).

[73] S. Sørensen and K. Mølmer, Quantum Computation with Ions in Thermal Motion, Phys. Rev. Lett. 82, 1971 (1999).

[74] J. D. Wong-Campos, S. A. Moses, K. G. Johnson, and C. Monroe, Demonstration of Two-Atom Entanglement with Ultrafast Optical Pulses, Phys. Rev. Lett. 119, 230501 (2017).

[75] V. M. Schäfer, C. J. Ballance, K. Thirumalai, L. J. Stephenson, T. G. Ballance, A. M. Steane, and D. M. Lucas, Fast quantum logic gates with trapped-ion qubits, Nature (London) 555, 75 (2018).

[76] L.-M. Duan, Scaling Ion Trap Quantum Computation through Fast Quantum Gates, Phys. Rev. Lett. 93, 100502 (2004).

[77] X.-C. Wu, Y. Ding, Y. Shi et al., ILP-based scheduling for linear-tape model trapped-ion quantum computers, in the Proceedings of The International Conference for High Performance Computing, Networking, Storage, and Analysis, Denver Co (2019).

[78] C. Y. Shih, S. Motlakunta, N. Kotibhaskar, M. Sajjan, R. Hablützel, and R. Islam, Reprogrammable and highprecision holographic optical addressing of trapped ions for scalable quantum control, npj Quantum Inf. 7, 57 (2021).

[79] Z. Mehdi, Z. K. Ratcliffe, and J. J. Hope, Fast entangling gates in long ion chains Phys. Rev. Research 3, 013026 (2021).

[80] Y.-K. Wu, Z.-D. Liu, W.-D. Zhao, and L.-M. Duan, Highfidelity entangling gates in a three-dimensional ion crystal under micromotion, Phys. Rev. A 103, 022419 (2021).

[81] S. Nagaitsev, A. Valishev, V. Danilov, and D. Shatilov, Design and simulation of IOTA-a novel concept of integrable optics test accelerator, in Part of Proceedings of the 3rd International Conference on Particle Accelerators, IPAC-2012, 1205201 (2012), pp. 16-19.

[82] R. S. Van Dyck, Jr., P. B. Schwinberg, and H. G. Dehmelt, Precise Measurements of Axial, Magnetron, Cyclotron, and Spin-Cyclotron-Beat Frequencies on an Isolated $1 \mathrm{meV}$ Electron, Phys. Rev. Lett. 38, 310 (1977).

[83] M. Fisher-Levine and A. Nomerotski, TimepixCam: A fast optical imager with time-stamping, J. Instrum. 11, C03016 (2016). 